\title{
Poly-L-lysine amplification of protamine immobilization and heparin adsorption
}

\author{
Yuehua Zhang, Vijendra K. Singh, Victor C. Yang \\ College of Pharmacy, University of Michigan, Ann Arbor, Michigan 48109-1065
}

Received 4 November 1997; accepted 18 March 1998

\begin{abstract}
We previously reported the development of a cellulose fiber based blood filter device containing immobilized protamine (termed protamine filter) that could be used to control both heparin- and protamine-induced complications during extracorporeal therapy. To achieve enhanced heparin adsorption on the fibers, we examined the possibility of utilizing the poly-L-lysine based amplification method to augment protamine loading on the fiber, as well as to create multiple layers of immobilized protamine for heparin interaction. Results show that such a method yielded about a threefold increase in protamine loading and, consequently,
\end{abstract}

about a fourfold enhancement in heparin adsorption when compared with the control without poly-L-lysine amplification. This technological improvement may facilitate development of a new generation of protamine filters with capacity and efficacy suitable for various clinical applications in extracorporeal heparin removal. (c) 1998 John Wiley \& Sons, Inc. J Biomed Mater Res, 42, 182-187, 1998.

Key words: protamine filter; chemical amplification; $\mathrm{CNBr}$ activation; protamine immobilization; heparin adsorption; poly-L-lysine conjugation

\section{INTRODUCTION}

Heparin, a highly sulfated glycosaminoglycan with an average molecular weight of $13 \mathrm{kDa}$, is commonly used as an anticoagulant drug during most surgical procedures, in particular extracorporeal blood circulation (ECBC). ${ }^{1,2}$ Systemic heparinization, however, results in a high incidence of bleeding complications. ${ }^{3,4}$ To prevent postoperative bleeding, protamine sulfate is routinely administered as a clinical heparin antagonist. ${ }^{5}$ Protamine is a low molecular weight $(4.5 \mathrm{kDa})$ protein that is rich in basic amino acids such as arginine. ${ }^{6}$ By virtue of its cationic nature, protamine interacts with the sulfate groups of heparin, thus neutralizing the anticoagulant activity of heparin. Unfortunately, the use of protamine can cause severe and at times fatal cardiovascular responses. ${ }^{7,8}$ Indeed, the administration of heparin and protamine has been suggested as the major cause of morbidity and mortality for patients undergoing cardiopulmonary bypass operations. ${ }^{9}$

We previously reported an approach that would simultaneously prevent heparin-induced and prot-

Correspondence to: Dr. Victor Yang; e-mail: vcyang@ umich.edu

Contract grant sponsor: NIH; Contract grant numbers: HL38353, HL55461

(c) 1998 John Wiley \& Sons, Inc.

CCC 0021-9304/98/020182-06 amine-induced complications. ${ }^{10}$ The approach consists of placing a hollow fiber based blood filter device containing immobilized protamine (called a protamine filter) at the distal end of the ECBC procedure. The protamine filter specifically removes heparin after heparin serves its anticoagulant purpose in the extracorporeal circuit, thereby eliminating heparin-induced bleeding risks. At the same time, the protamine filter does not allow the protamine to enter the patient, thereby minimizing protamine-induced toxic effects. Previous in vitro ${ }^{1}$ and in vivo studies ${ }^{12}$ demonstrated the feasibility and utility of the approach. Using the dog as an animal model and at a blood flow of 100 $\mathrm{mL} / \mathrm{min}$, the protamine filter removed more than $60 \%$ of the administered heparin in $10 \mathrm{~min} .{ }^{12}$ While rapid protamine injection produced profound hypotensive responses in dogs, the use of the protamine filter did not elicit any significant change in hemodynamic parameters. ${ }^{12}$

The bottleneck limitation in heparin removal by the protamine filter lies in its lack of capacity, because protamine loading and heparin adsorption on the filter are both restricted by the surface area of the hollow fibers. This was reflected in the in vivo studies in which the filter was saturated within $10 \mathrm{~min}$ of operation. ${ }^{12}$ In the study presented here we therefore explored the possibility of utilizing a chemical amplification method reported by Lin and coworkers ${ }^{13}$ to enhance protamine loading and create multiple immobilized protamine layers on the filter for heparin 
adsorption. Poly-L-lysine was selected as the chemical amplifier, and the effects of its molecular weight on the amplification process was also examined.

\section{MATERIALS AND METHODS}

\section{Reagents and instruments}

Cellulose hollow fibers were obtained by disassembling a Travenol (Deerfield, IL) model 1500 CF hemodialyzer. Each fiber has an internal diameter of $200 \mu \mathrm{m}$ and a wall thickness of $10 \mu \mathrm{m}$ and is made of Cuprophan membrane with a molecular weight cutoff of $10 \mathrm{kDa}$. Herring protamine sulfate (grade III) was purchased from Sigma (St. Louis, MO). Porcine intestinal mucosa heparin (sodium salt, 150 USP U/mg) was purchased from Pharmacia-Hepar Industries (Franklin, $\mathrm{OH})$. Cyanogen bromide (CNBr), poly-L-lysine, and other chemicals were purchased from Aldrich Chemical Co. (Milwaukee, WI). All aqueous solutions were prepared in deionized distilled water. IR spectra ( $\mathrm{KBr}$ pellets) were recorded on a Nicolet DX V4.56 FTIR spectrometer (Nicolet, Madison, WI), and UV-VIS spectra were measured with a Lambda 7 UV/VIS spectrophotometer (Perkin-Elmer, Uberlingen, Germany). The potentiometric response of the heparin and protamine membrane electrode was measured with a $\mathrm{pH}$ meter (Fisher Scientific Accumet model 25).

\section{Activation of fiber particles}

Fine hollow fiber particles were prepared and activated according to the procedures described previously. ${ }^{11}$ In brief, the hollow fiber was chopped into smaller fragments (about $200 \mathrm{~mm}$ long), frozen in liquid nitrogen, ground in a crucible, and then passed through molecular sieves (pore size, 75 and $90 \mu \mathrm{m})$. To activate the fibers, $0.5 \mathrm{~g}$ of the fiber particles were suspended in $2.5 \mathrm{~mL}$ of distilled water and stirred for $1 \mathrm{~h}$ at $0-5^{\circ} \mathrm{C}$. Two and half milliliters of acetone containing different amounts of $\mathrm{CNBr}$ were then added to the vigorously stirred suspension, followed by the dropwise addition of triethylamine at a molar ratio of 1:1.5 of CNBr to triethylamine. The reaction was continued for $20 \mathrm{~min}$ at $4^{\circ} \mathrm{C}$ under vigorous agitation. The activated fiber particles were then filtered, washed with ice-cold 50\% acetone, and rinsed several times with ice-cold distilled water. The cyanate ester content of activated fiber particles was determined using the colorimetric method described by Kohn and Wilchek. ${ }^{14}$

\section{Coupling of poly-L-lysine to fiber particles}

Poly-L-lysine hydrobromide, dissolved in coupling buffer (0.2M NaHCO $3,0.5 \mathrm{M} \mathrm{NaCl}, \mathrm{pH} 9.0)$, was mixed with the $\mathrm{CNBr}$-activated fiber particles $(0.2 \mathrm{~g})$ prepared above. The suspension was agitated for $4 \mathrm{~h}$ at room temperature. The fiber particles were then washed sequentially with the cou- pling buffer of $0.25 \mathrm{M}$ phosphate buffer containing $0.5 \mathrm{M}$ $\mathrm{NaCl}, \mathrm{pH}$ 7.0. The amount of poly-L-lysine coupled to the fiber particles was determined by acid-base titrations. ${ }^{15}$

\section{Protamine immobilization to poly-L-lysine linked fiber particles}

The poly-L-lysine conjugated fiber particles were activated with $\mathrm{CNBr}$, using the same procedures described above. Afterward, the fiber particles were suspended in $2 \mathrm{~mL}$ of protamine solution $(10 \mathrm{mg} / \mathrm{mL})$ in sodium bicarbonate buffer $\left(0.1 \mathrm{M} \mathrm{NaHCO}_{3}, 0.15 \mathrm{M} \mathrm{NaCl}, \mathrm{pH} 8.3\right)$ and then agitated at room temperature for $20 \mathrm{~h}$. After filtration under the vacuum, the fiber particles was washed thoroughly with $0.25 \mathrm{M}$ phosphate buffer ( $\mathrm{pH} 7.0$ ) containing $0.15 \mathrm{M} \mathrm{NaCl}$, followed by rinsing with distilled water several times. The amount of protamine immobilized on the fiber particles was estimated by measuring the protamine concentration in the coupling solution before and after immobilization, as well as in the washing solutions. Protamine concentration was determined by titration using the protamine sensor method developed previously in our laboratory. ${ }^{16}$

\section{Heparin adsorption on protamine-linked fiber particles}

The protamine-immobilized fiber particles $(0.2 \mathrm{~g})$ were suspended in $2 \mathrm{~mL}$ of heparin solution $(10 \mathrm{mg} / \mathrm{mL})$. The suspension, after mixing for $2 \mathrm{~h}$ at room temperature, was filtered under a vacuum. The fiber particles were washed with $20 \mathrm{~mL}$ of $0.15 \mathrm{M} \mathrm{NaCl}$ followed by $50 \mathrm{~mL}$ of distilled water. The amount of heparin adsorbed on the protaminelinked fiber particles was estimated by measuring the heparin concentration in the solution before and after adsorption, as well as in the washing solutions. Heparin concentration was determined by titration using the protamine sensor method developed previously in our laboratory. ${ }^{16}$

\section{RESULTS AND DISCUSSION}

In an attempt to increase the amount of immobilized heparin on a biomaterial surface, Lin and coworkers ${ }^{13}$ reported a novel chemical amplification method that employed high molecular weight (50-100 kDa) polyethyleneimine (PEI) as the amplifying agent. Their results showed that after grafting the polyurethane surface with the polyfunctional PEI compound, the reactive sites on the polyurethane surface for subsequent heparin immobilization was increased by about sevenfold whereas the total amount of heparin immobilized on the PEI-amplified surface was improved nearly twofold. Because the primary limitation of our heparin removing system is also related to the capacity of the protamine filter, we examined the possibility 
of utilizing this amplification method to improve protamine loading on the fibers, as well as to create multiple immobilized protamine layers for heparin interaction. A schematic diagram of the principle of our approach is illustrated in Figure 1. Poly-L-lysine was selected as the amplifying agent because it possesses excessive $-\mathrm{NH}_{2}$ functional groups for activation and subsequent protamine immobilization. As depicted in Figure 1, the approach consists of four steps: (i) activation of the hydroxyl groups on cellulose fibers, (ii)

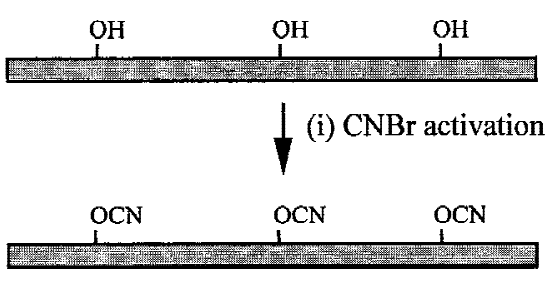

(ii) Poly-L-lysine coupling

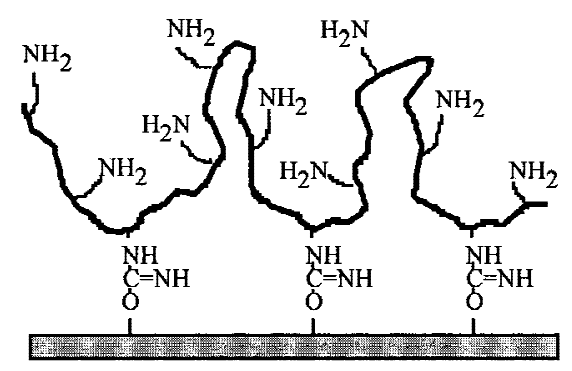

(iii) $\mathrm{CNBr}$ activation
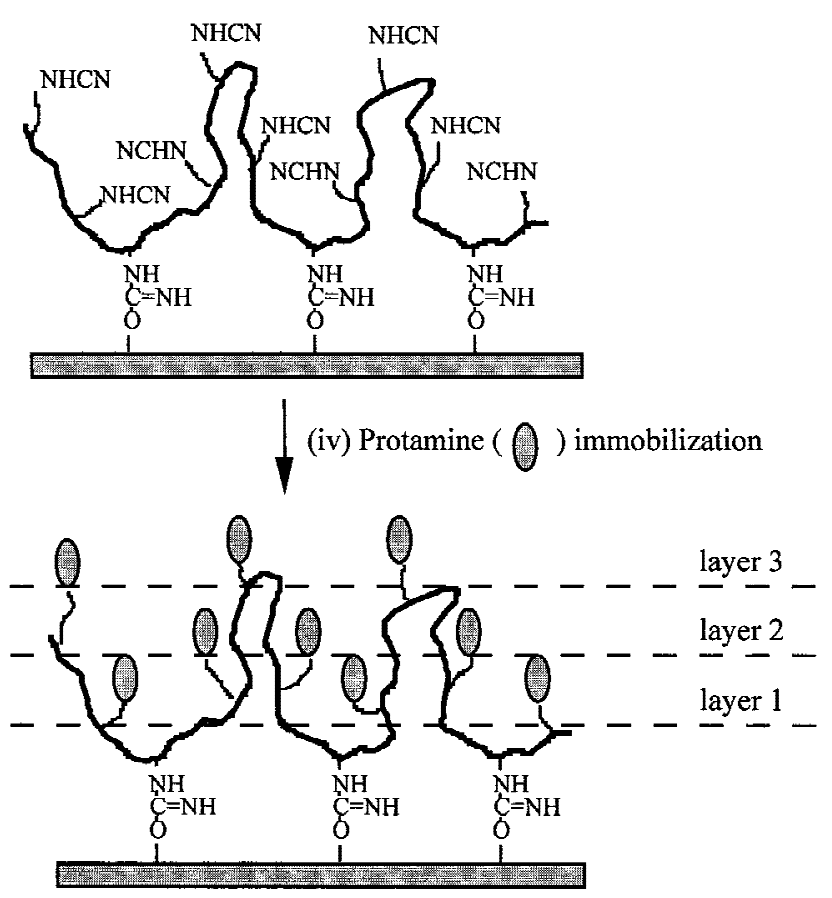

Figure 1. Schematic diagram of polymer surface modification with poly-L-lysine for enhanced protamine immobilization and heparin adsorption. covalent coupling of poly-L-lysine to activated cellulose fibers, (iii) activation of the primary amino groups of immobilized poly-L-lysine, and (iv) covalent coupling of protamine to activated poly-L-lysine. $\mathrm{CNBr}$ was selected as the activation reagent because it activates hydroxyl groups that are abundant in cellulose fibers. The CNBr activation method described by Kohn and Wilchek ${ }^{17}$ was followed, because this method is known to improve the yield of activated cyanate ester groups by reducing their hydrolysis. Indeed, by using low amounts of CNBr $(0.25-2 \mathrm{mmol} /$ $\mathrm{mL}$ ), a linear increase of cyanate ester formation was observed (data not shown). The reaction was linear up to $20 \mathrm{~min}$, a time interval that yielded optimal cyanate ester content $(156 \mu \mathrm{mol} / \mathrm{g})$. Thus, a reaction time of 20 min was chosen for subsequent studies.

The Cuprophan dialysis membrane employed in this study has a molecular weight cutoff of $10 \mathrm{kDa}$. For protamine and heparin with a respective average molecular weight of 4.5 and $13 \mathrm{kDa}$, it is likely that both protamine immobilization and subsequent heparin adsorption are affected by the external (i.e., diffusion of the molecules from the aqueous phase to the surface of the cellulose fibers), as well as internal (i.e., diffusion of the molecules within the fiber matrices) diffusion limits. To precisely evaluate the amplification effects of poly-L-lysine, ground fibers were used to eliminate the internal diffusion limits and a stirring system was used to eliminate the external diffusion limits. The feasibility and utility of such approaches in eliminating diffusion limits were demonstrated in our previous work. ${ }^{18}$

During each step of the solid phase syntheses, the reaction products were characterized by IR spectrometric analysis (Fig. 2). When compared to untreated cellulose fiber (spectrum [0]), the CNBr-activated fiber (spectrum [i]) showed two new absorption bands: one at $2256 \mathrm{~cm}^{-1}$ that was attributed to the added $-\mathrm{CN}$ groups, and the other one at $1700 \mathrm{~cm}^{-1}$ that was attributed to the $\mathrm{C}=\mathrm{O}$ groups of the amide bonds resulting from hydrolysis of the cyanate ester $-\mathrm{O}-\mathrm{CN}$ ) groups [see step (i) in Fig. 1 for the chemical reaction]. Following poly-L-lysine immobilization (spectrum [ii]), the band at the $2256 \mathrm{~cm}^{-1}$ wavelength disappeared, which suggested the coupling of poly-L-lysine via the activated $-\mathrm{O}-\mathrm{CN}$ groups. On the other hand, the intensity of the band at $1700 \mathrm{~cm}^{-1}$ increased significantly and, indeed, overlapped with the $\mathrm{H}_{2} \mathrm{O}$ band at $1650 \mathrm{~cm}^{-1}$ seen in the untreated fibers (see spectrum [0]). This change was primarily due to the presence of a large number of amide bonds in the immobilized poly-L-lysine. When the poly-L-lysine linked fibers were activated again with $\mathrm{CNBr}$ (spectrum [iii]), the absorption band at $2256 \mathrm{~cm}^{-1}$ reappeared, indicating new incorporation of $-\mathrm{CN}$ groups to the fiber surface. After coupling of protamine to immobilized poly-Llysine on the fibers via these - CN groups, the absorp- 


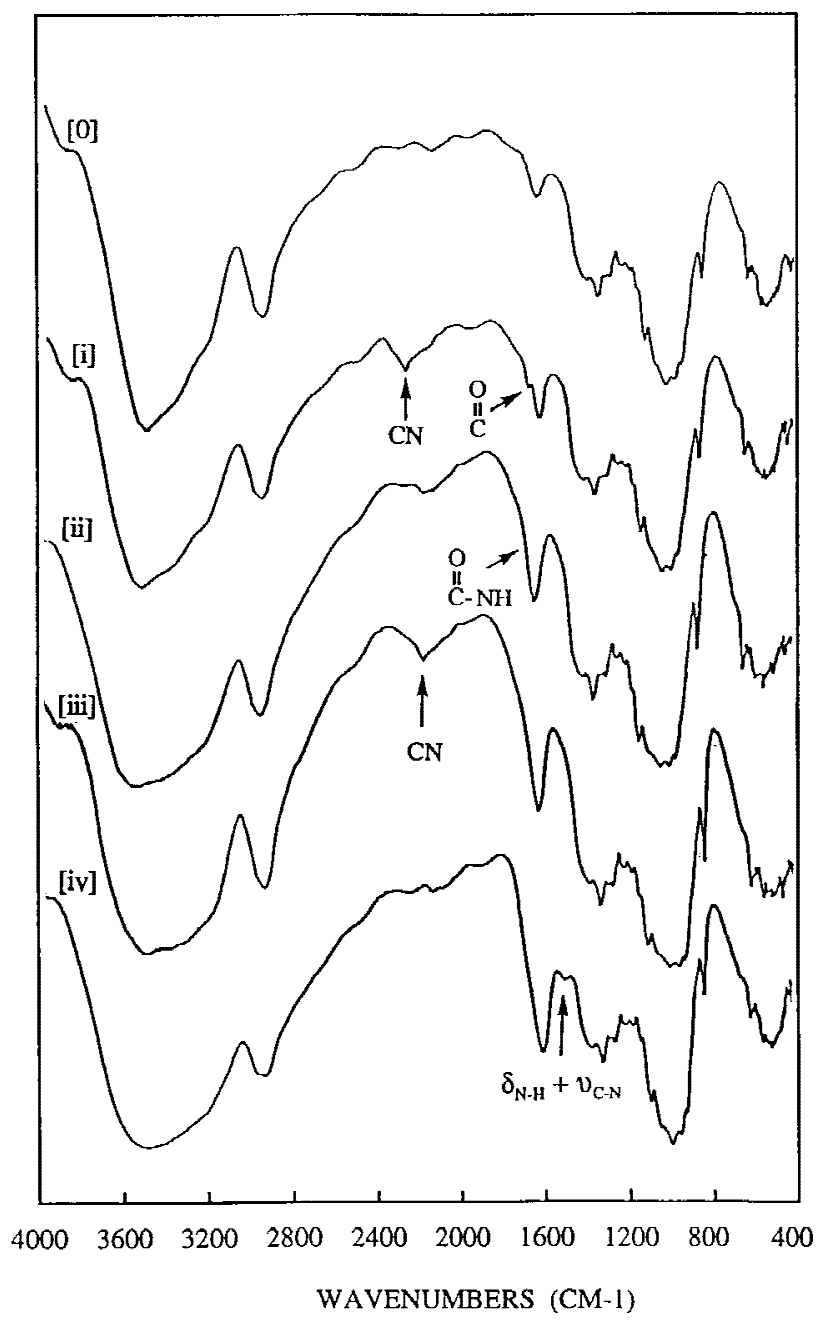

Figure 2. IR spectra of polymer fiber at various steps of chemical modification. (0) cellulose fiber, (i) $\mathrm{CNBr}$ activated fiber, (ii) poly-L-lysine conjugated fiber, (iii) CNBr-activated poly-L-lysine fiber, and (iv) protamine-immobilized fiber.

tion band at $2256 \mathrm{~cm}^{-1}$ disappeared (spectrum [iv]). It is interesting to note that a new band at $1539 \mathrm{~cm}^{-1}$ $\left(\delta_{\mathrm{N}-\mathrm{H}}+v_{\mathrm{C}-\mathrm{N}}\right)$ appeared. The occurrence of this relatively weak band may be attributed to a significant increase in stretching $(\delta)$ and bending $(v)$ vibrations of the $\mathrm{C}-\mathrm{N}$ and $\mathrm{N}-\mathrm{H}$ bonds on the fiber surface, presumably due to the presence of a large number of amide bonds in the newly immobilized protamine molecules.

The effect of the molecular weight of poly-L-lysine on the amplification of protamine immobilization and heparin adsorption on cellulose fibers was examined. Three poly-L-lysine species were selected that possessed molecular weights of 2.5, 22.5, and $110 \mathrm{kDa}$. This selection was by design to ensure that one molecular weight was below, one was close to, and one was significantly higher than that of heparin (average molecular weight, $13 \mathrm{kDa}$ ). Heparin, protamine, and poly-L-lysine are all known to be relatively linear molecules due to their high density of repelling charges.
Thus, theoretically it would require at least a molecular weight (or length) of heparin for poly-L-lysine to enable the creation of an additional surface layer for heparin adsorption. The protamine loading and heparin adsorption data are summarized in Table I. It should be pointed out that although poly-L-lysine is known to bind heparin, it would yield, at best, a minimal effect on the enhanced heparin adsorption. This is simply because all the $-\mathrm{NH}_{2}$ groups on poly-L-lysine, which are involved in heparin binding, have been activated by $\mathrm{CNBr}$ and then linked to protamine via guanidino linkages or then converted to ureido groups due to possible hydrolysis of the guanidino linkages. Table I shows that for all of the three polyL-lysine species studied, the protamine loading increased with increasing molar ratios of poly-L-lysine/ $\mathrm{CNBr}$ (during amplification) up to a point and plateaued thereafter with a further increase of the ratio. (Although data were not shown in the experiments with the 110-kDa poly-L-lysine as the amplifier, protamine loading remained approximately $36 \mathrm{mg} / \mathrm{g}$ fiber when the poly-L-lysine/CNBr molar ratio was further increased to 0.40.) Such an increase was related, to some degree, to the addition of poly-L-lysine based reactive sites for protamine immobilization. Alternatively, the protamine loading and, consequently, the heparin adsorption, also increased with increasing molecular weight of poly-L-lysine, suggesting the amplifier effect of poly-L-lysine and multiple protamine layer formation for heparin adsorption. Interestingly, protamine loading on fibers amplified with the 2.5$\mathrm{kDa}$ poly-L-lysine (whose molecular weight was presumably not sufficient enough to create a second layer of immobilized protamine) was markedly increased by twofold over the control when no poly-L-lysine was

TABLE I

Protamine Loading and Heparin Adsorption Capacity of Chemically Modified Cellulose Fiber

\begin{tabular}{ccccc}
\hline $\begin{array}{c}\text { Fiber } \\
\text { No. }\end{array}$ & $\begin{array}{c}\text { Poly-L- } \\
\text { Lysine } \\
\text { (kDa) }\end{array}$ & $\begin{array}{c}\text { Poly-L-Lysine } \\
\text { to CN Group } \\
\text { (Mole Ratio) }\end{array}$ & $\begin{array}{c}\text { Protamine } \\
\text { Loading } \\
\text { (mg/g Fiber) }\end{array}$ & $\begin{array}{c}\text { Heparin } \\
\text { Adsorption } \\
\text { (mg/g Fiber) }\end{array}$ \\
\hline 1 & None & None & 13.5 & 9.1 \\
2 & 2.5 & 0.3 & 17.8 & 10.8 \\
3 & 2.5 & 0.6 & 20.2 & 12.9 \\
4 & 2.5 & 0.8 & 21.8 & 13.5 \\
5 & 2.5 & 1.2 & 25.0 & 15.5 \\
6 & 2.5 & 1.5 & 27.6 & 21.0 \\
7 & 2.5 & 2.0 & 29.0 & 24.0 \\
8 & 22.5 & 0.2 & 20.5 & 13.4 \\
9 & 22.5 & 0.4 & 29.0 & 25.0 \\
10 & 22.5 & 0.6 & 33.1 & 28.0 \\
11 & 22.5 & 0.8 & 34.0 & 29.0 \\
12 & 110 & 0.1 & 19.6 & 14.2 \\
13 & 110 & 0.2 & 27.0 & 25.0 \\
14 & 110 & 0.25 & 31.4 & 32.0 \\
15 & 110 & 0.35 & 36.4 & 38.0 \\
\hline
\end{tabular}


used (i.e., from 13.5 to $29 \mathrm{mg}$ protamine/g fiber; see Table I). All of these findings can be accounted for in terms of a hypothetical mechanism derived for the amplification process (Fig. 3). When no poly-L-lysine was used (i.e., the control, sample 1 in Table I), protamine was immobilized directly on the fiber surface and immobilized protamine was arrayed in a brushlike structure. Consequently, heparin adsorption on these immobilized protamine molecules was 2dimensional and was controlled by the total surface area of the fibers [see Fig. 3(A)]. When the $2.5-\mathrm{kDa}$ poly-L-lysine was used as the amplifier (samples 2-7), although this molecular weight was not sufficient to create a second layer of immobilized protamine for heparin interaction, it nevertheless functioned like the truncal part of a pine tree, to which protamine was immobilized like all the pine needles. Protamine immobilization and heparin adsorption to such immobi- lized protamine molecules therefore became 3dimensional and were significantly augmented over that of the control due to a more spacious arrangement [see Fig. 3(B)]. When the molecular weight of poly-Llysine was raised to that of heparin (samples 8-11) or significantly above it (samples 12-15), either double or multiple layers of immobilized protamine, respectively, were formed. The amplification by poly-Llysine on protamine loading and subsequent heparin adsorption in this case was a combination of the 3dimensional immobilization of protamine molecules and the presence of multiple immobilized protamine layers for heparin interaction [see Fig. 3(C)]. Further analysis of the data in Table I yields support to this hypothesis. When the protamine loading ( $x$ axis) was plotted against the amount of heparin adsorption ( $y$ axis) for each of three poly-L-lysine species examined, the slopes were 1.13,1.16, and $1.43 \mathrm{~g} / \mathrm{g}$ (heparin/

(A)
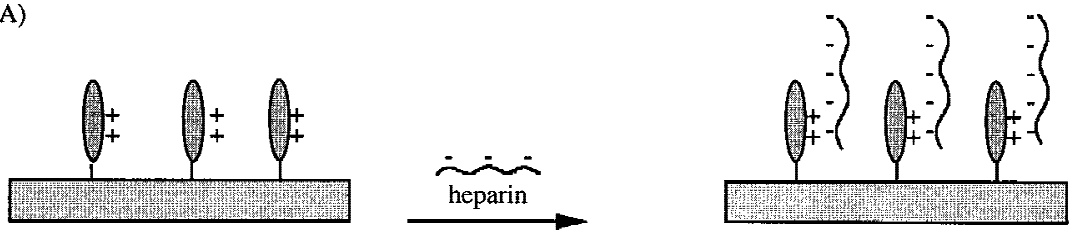

(B)

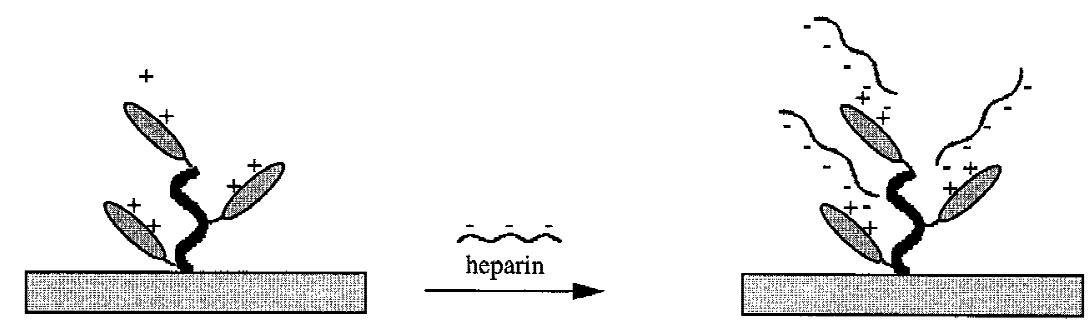

(C)

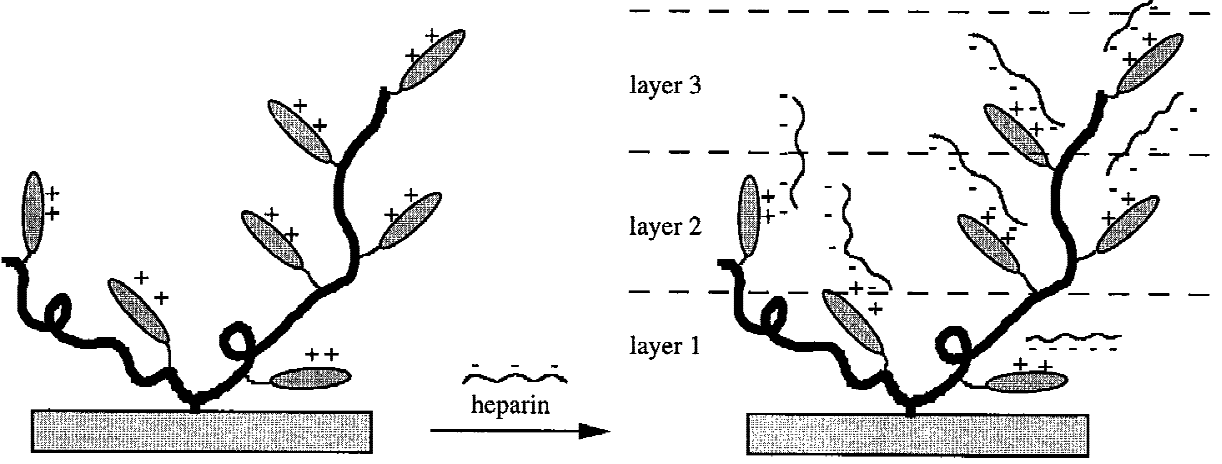

: cellulose fiber; : protamine; 2

Figure 3. Hypothetical mechanism of heparin adsorption on protamine-immobilized fibers. (A) Fiber without poly-L-lysine, (B) fiber with low molecular weight poly-L-lysine, and (C) fiber with high molecular weight poly-L-lysine. 
protamine) for the $2.5,22.5$, and $110 \mathrm{kDa}$ poly-L-lysine species, respectively. It should be pointed out that the slope represents the specific activity of immobilized protamine in heparin adsorption. Because all the polyL-lysine species carry the same density and distribution of reactive groups (i.e., the $-\mathrm{NH}_{2}$ group) for protamine immobilization, regardless of their molecular weights, protamine immobilized on different poly-Llysine species should possess the same specific activity in heparin adsorption, as long as they are under the same environment (such as within the same surface layer). The significant increase in specific activity using $110-\mathrm{kDa}$ poly-L-lysine relative to $2.5-\mathrm{kDa}$ species strongly implicates the formation of more than one layer of immobilized protamine, simply because under this situation the immobilized protamine molecules are more capable of interacting with heparin due to their spacious arrangement and significantly enhanced freedom for dynamic motion. On the other hand, the slight increase in specific activity from 2.5 to $22.5 \mathrm{kDa}$ poly-L-lysine species suggests that the latter still does not possess sufficient molecular chain length to produce a thorough second layer of immobilized protamine for heparin adsorption.

In summary, the feasibility of utilizing a poly-Llysine based amplification method to promote protamine immobilization and consequently heparin adsorption has been demonstrated. By adapting an optimized procedure and an appropriate molecular weight for poly-L-lysine, the overall protamine loading on the cellulose fibers was enhanced 2.7-fold, which also resulted in over a fourfold increase of heparin adsorption on the fiber. This observation may potentially lead to development of a highly effective protamine filter for the clinical removal of heparin. In this respect, further studies are currently in progress in our laboratories to ultimately design a new generation of protamine filters that will have clinically acceptable size (e.g., smaller than that of a hemodialyzer cartridge), capacity, and efficacy to remove heparin under the most demanded heparin doses in extracorporeal procedures such as cardiopulmonary bypass. The amplification method described herein can also be applied to enhance the efficiency of most affinity-type chromatographic methods, as well as to augment the capacity for capturing analytes of most solid-state immunoassays.

\section{References}

1. I. Danishefsky, L. Rosenfeld, L. Kuhn, B. Lahiri, and C. Whyzmuzis, "Heparin and related polysaccharides, structure and activities," Ann. NY Acad. Sci., 556, 29-36 (1989).

2. L. B. Jaques, "Heparin: Anionic polyelectrolyte drugs," Pharmacol. Rev., 31, 99-166 (1980).

3. A. G. Gavin, "Complications of heparin therapy," Surg. Gynecol. Obstet., 140, 789-796 (1975).

4. M. N. Levine and J. Hirsch, "Hemorrhagic complications of anticoagulant therapy," Semin. Thromb. Hemostasis, 12, 39-57 (1985).

5. L. B. Jaques, "Protamine: Antagonist to heparin," Can. Med. Assoc. J., 108, 1291-1297 (1973).

6. T. Ando, M. Yamsaki, K. Suzuki, "Protamine," in Molecular Biology Biochemistry and Biophysics, A. Kleinzeller (ed.), Springer-Verlag, New York, 1973, pp. 1-109.

7. C. A. Cobb III and D. L. Fung, "Shock due to protamine hypersensitivity," Surg. Neurol., 17, 245-246 (1982).

8. M. D. Sharath, W. J. Metzger, H. B. Richerson, R. K. Scupham, R. L. Meng, B. H. Ginsberg, and J. M. Weiler, "Protamine induced fatal anaphylaxis," J. Thorac. Cardiovasc. Surg., 90, 86-90 (1985).

9. J. M. Weiler, M. A. Gellhaus, J. G. Carter, et al., "A prospective study of the risk of an immediate adverse reaction to protamine sulfate during cardiopulmonary bypass surgery," $\mathrm{J}$. Allergy Clin. Immunol., 85, 713-719 (1990).

10. V. C. Yang, "Extracorporeal blood deheparinization system," U.S. Pat. 4,800,016 (1987)

11. J. S. Kim, A. J. Yang, and V. C. Yang, "Protamine immobilization and heparin adsorption on the protamine-bound cellulose fiber membrane," Biotechnol. Bioeng., 39, 450-456 (1992).

12. V. C. Yang, F. K. Port, J. S. Kim, C. L. Teng, G. O. Till, and T. W. Wakefield, "The use of immobilization protamine in removing heparin and preventing protamine-induced complications during extracorporeal blood circulation," Anesthesiology, 75, 288-297 (1991).

13. S. C. Lin, H. A. Jacobs, and S. W. Kim, "Heparin immobilization increased through chemical amplification," J. Biomed. Mater. Res., 25, 791-795 (1991).

14. J. Kohn and M. Wilchek, "A colorimetric method for monitoring activation of sepharose by cyanogen bromide," Biochem. Biophys. Res. Commun., 84, 7-14 (1978).

15. Z. J. Wang, "Determination of the amino group in chitosan by alkalimetry," Huaxue Shijie, 27, 22-23 (1986).

16. J. H. Yun, M. E. Meyerhoff, and V. C. Yang, "Protaminesensitive polymer membrane electrode: Characterization and bioanalytical applications," Anal. Biochem., 224, 212-220 (1995).

17. J. Kohn and M. Wilchek, "A new approach (cyano-transfer) for cyanogen bromide activation of sepharose at neutral $\mathrm{pH}$, which yields activated resins, free of interfering nitrogen derivatives," Biochem. Biophys. Res. Commun., 107, 878-884 (1992).

18. H. Bernstein, V. C. Yang, and R. Langer, "The distribution of heparinase covalently immobilized to agarose: Experimental and theoretical studies," Biotechnol. Bioeng., 30, 197-207 (1987). 